\title{
Tibial torsion analysis in computed tomography: development and validation of a real 3D measurement technique
}

\author{
Armando Hoch ${ }^{1,2^{*}+} \mathbb{0}$, Tabitha Roth ${ }^{2 \dagger}$, Magda Marcon ${ }^{3}$, Philipp Fürnstahl ${ }^{2}$, Sandro F. Fucentese ${ }^{1}$ \\ and Reto Sutter ${ }^{3}$
}

\begin{abstract}
Purpose: Pathological tibial torsion is known to negatively influence the functionality of the lower extremity, and therefore, its assessment might play an important role. While 3D imaging is used for many examinations of the musculoskeletal system, for the determination of tibial torsion no 3D measurement technique has been available so far. We developed a 3D measurement method and assess its interobserver reliability as well as its correlation with standard 2D measurement methods.
\end{abstract}

Methods: CT scans of 82 tibiae in 79 patients with a mean age of 41 years were included. A novel 3D measurement technique was developed and applied. Measurements were compared with two frequently used 2D measurement methods. ICC (intraclass correlation coefficient) for the new technique was determined and compared to the 2D measurement method. Furthermore, differences between left and right legs as well as between males and females were assessed.

Results: The ICC for the 2D methods was 0.917 and 0.938, respectively. For the 3D measurements, ICCs were calculated to be 0.954 and 0.950. Agreement between 2 and 3D methods was moderate to good with ICCs between 0.715 and 0.795. Torsion values for left and right legs did not differ significantly in $2 \mathrm{D}$ and in $3 \mathrm{D}\left(26.2 \mathrm{vs} 28.5^{\circ}\right.$ and $27.2 \mathrm{vs}$. $25.9^{\circ}$ ). The same is true for the differences between male and female in 2D and 3D ( $26.2 \mathrm{vs.} 29.6^{\circ}$ and $25.0 \mathrm{vs} .31 .2^{\circ}$ ).

Conclusion: The newly developed 3D measurement technique shows a high intraclass agreement and offers an applicable opportunity to assess the tibial torsion three-dimensionally.

Keywords: Tibial torsion, 3D measurement, Lower-limb deformity, Computed tomography, Torsional malalignment

\section{Key messages}

- The tibial torsions relevance in maltorsion of the lower extremity and respective surgical planning is underestimated.

- So far, no real 3D measurement technique in sectional imaging was elaborated.

\footnotetext{
*Correspondence: armando.hoch@balgrist.ch

${ }^{+}$Armando Hoch and Tabitha Roth contributed equally to this work

1 Department of Orthopaedics, Balgrist University Hospital, University

of Zurich, Forchstrasse 340, 8008 Zurich, Switzerland

Full list of author information is available at the end of the article
}

- Our novel 3D measurement technique showed an excellent intraclass correlation.

\section{Introduction}

Rotation of the tibia along its longitudinal axis was first described in an anatomical study in 1903 by Pierre Germain Marie Le Damany, who was a professor for anatomy and physiology, famous for his early work on the developmental dysplasia of the hip (DDH) [1]. According to him, excessive tibial torsion can be understoodlike DDH-as element of the 'packing disorders' that can arise during the development and growth of the 
fetus in utero. Elements of his pioneering work remain true to this day [1-3]. The term 'tibial torsion' was then consolidated in a radiographic study in 1949 [4]. Since the tibial torsion is subject to major changes during childhood, most studies have focused on its normal and pathological development in this early phase of life [5-10]. Decreased or increased tibial torsion was found to be associated with the clinical picture of in- or outtoeing, which in turn is associated with a higher risk for stumbling, gait abnormalities and aesthetic complaints $[7,8,10]$. In adults, the tibial torsion can be pathologically altered after trauma and consecutive surgery (e.g., intramedullary nails), sometimes leading to an inferior clinical outcome [11-15], such as a reduced capacity of muscles to extend the hip and knee joints in patients with excessive tibial torsion [16]. Furthermore, rotational deformities of the lower extremity in general are associated with a higher risk for osteoarthritis of the involved joints [17-21]. Surgical correction due to these issues is sometimes necessary, and therefore, a validated measurement method for the tibial torsion is required. Radiographic measurement methods using standard radiographs [4,9] or fluoroscopy [22] have proven to be inadequate, since their reproducibility is low. With the emerging possibilities in cross-sectional imaging, different computed tomography (CT)-based 2D measurement methods were proposed [23-28]. In contrast to the femur however, a clear definition of the joint axes is difficult for the tibia. On the one hand, the determination of the proximal axis for 2D measurements, the highly variable posterior shape of the proximal epiphysis and metaphysis is a challenge for routine measurements. On the other hand, the distal axis, as defined by most standard methods, is directly dependent on the highly variable position of the fibula [24]. Various attempts have been undertaken to assess 2D measurements of tibial torsion, but the variabilities between some of these methods remain substantial [23-28]. Thus, the role of the tibial torsion in lower extremity deformities remains unclear, and with the increasing demand of a holistic 3D deformity analysis, a reliable measurement technique for the tibial torsion is mandatory. To our knowledge, there is no measurement technique which considers the three-dimensional (3D) constitution of the tibia using sectional imaging, and also the relevance of the position of the fibula for calculation of the tibial torsion has never been evaluated. Therefore, the first purpose of this study was to develop and validate a 3D measurement technique to assess tibial torsion. The second purpose was to investigate the correlation between by the most frequently used 2D measurement methods with the newly developed 3D technique.

\section{Material and methods}

\section{Patient selection}

We retrospectively identified 79 consecutive patients who received a CT scan of one or both legs. The data acquisition was performed using the MyOsteotomy CT protocol (Medacta, Castel San Pietro, Switzerland) in which only the hip, knee and ankle joints were scanned, while skipping midshaft regions. All CT scans were performed at our institution between 03/2016 and 12/2018. These patients obtained the CT scan in the scope of the preoperative planning for lower-limb surgical procedures (high tibial osteotomy in unicompartmental osteoarthritis of the knee) using patient-specific instruments. Three patients with prior osseous surgery on the tibia were excluded subsequently in order to obtain a collective without iatrogenic alteration of bony anatomy. Finally, we included CT scans of 82 knees from 79 patients (57 males and 22 females, 49 left and 33 right legs) with a mean age of 41 years (range 15-64 years). The local ethical committee approved this study (Zurich Cantonal Ethics Commission, 2017-01,616), and all patients gave their informed consent for the use of their data for research purposes.

\section{$\mathrm{CT}$ examinations and segmentation}

All CT scans were performed at our institution, using a 64-detector row CT scanner (Somatom Definition AS Siemens Healthcare, Erlangen, Germany). Slice thickness was $1.0 \mathrm{~mm}$ with an in-plane resolution of $0.4 \times 0.4 \mathrm{~mm}$. The CT scans were segmented using the global thresholding and region growing functionality of a standard segmentation software (Mimics Medical, Materialise NV, Leuven, Belgium) in order to generate 3D bone models [29-31].

\section{D measurement methods}

Tibial torsion in 3D was defined as the angle between the proximal (PTA) and the distal tibia axis (DTA), projected to the plane perpendicular to the anatomical axis of the tibia (Fig. 1). While the PTA was the same for all measurements in one case, the DTA was calculated twice for each bone: once by considering only the distal tibia itself (3D-T) and once by additionally including the distal fibula (3D-TF).

Prior to the definition of the PTA and DTA, the bone models have to be aligned in a reference coordinate system. The mechanical axis, determined as described by Fürnstahl et al. [32], was aligned with the y-axis, and the leg was rotated until the patella was facing anteriorly.

The PTA was defined by a line connecting the points on the tibial plateau that are closest to the most distal tips of the femoral condyles. In a first step, the two condyles were automatically detected by applying a k-means 


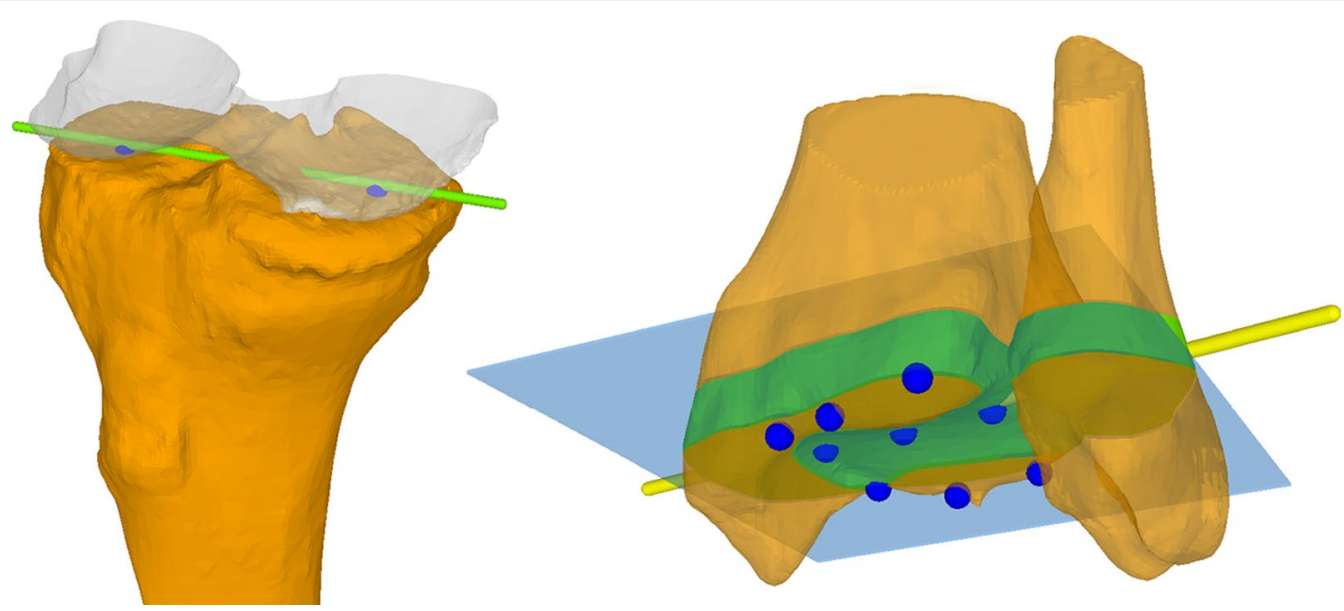

Fig. 1 Definition of the proximal and distal tibia axes in 3D shown on a model of the proximal tibia (orange) and distal femur (transparent grey) on the left and the distal tibia and fibula (both orange) on the right side. Left: the most distal points were identified for each femoral condyle, and PCA was applied to the corresponding points on the tibial plateau (blue dots). The PTA was defined as the first principle component. Right: A plane (DTJP, blue) was fitted to nine manually selected points on the articular surface (blue dots). A bone slice of $10 \mathrm{~mm}$ thickness was extracted distal from the DTJP (green), and PCA was applied to find the DTA (yellow line). DTA was defined as the second principle component. The DTA was calculated in two different adaptations (with and without consideration of the fibula). PCA principle component analysis, PTA proximal tibia axis, DTJP distal tibia joint plane, DTA distal tibia axis

algorithm [33] to the most distal $25 \mathrm{~mm}$ of the femoral bone, initiated as described by Arthur et al. [34]. Two condylar tip clusters (CTC) were defined as all points within the most distal $1 \mathrm{~mm}$ of each condyle. Subsequently, the closest point on the tibial plateau was found for each point within the CTCs, yielding the tibiofemoral contact areas (TFCA) on the tibial plateau. Finally, principal component analysis (PCA) was applied to the TFCA, defining the PTA [35].

In order to determine the DTA, the distal tibia joint plane (DTJP) was fitted to the articular surface of the distal tibia by applying a least square plane fit algorithm to nine manually selected points (Fig. 1) [35]. We then isolated the $10 \mathrm{~mm}$ of tibial metaphysis located directly proximal of the DTJP and used it to define the DTA by applying PCA [35]. For the determination of the 3D-TF tibial torsion, the corresponding $10 \mathrm{~mm}$ of fibular metaphysis was additionally included.

Both PTA and DTA were finally projected onto a plane perpendicular to the anatomical axis of the tibia. The anatomical axis was defined by fitting a line through the center points of six intersections along the tibia.

These points for the anatomical axis were found by first fitting a plane to the tibial plateau by applying a least square fit algorithm [35] to eight manually selected points on the plateau, resulting in the tibial plateau plane (TPP). Thereafter, PCA was applied to the whole tibial bone. The first principal component was used as the plane normal for six parallel planes placed along the tibial metaphysis and proximal shaft in $20-\mathrm{mm}$ intervals, starting $40 \mathrm{~mm}$ distal from the center of the TPP. For each of these planes, the intersection with the tibial bone was calculated, and the center point of each intersection was determined. Finally, the anatomical axis was fit through these six center points.

\section{D measurement methods}

Two frequently used 2D measurement techniques were chosen for comparison to the proposed 3D technique (Fig. 2). Both techniques used the same approach to determine the orientation of the PTA. A tangent was placed along the posterior cortex of the tibial head one slice $(1.0 \mathrm{~mm})$ proximal to the fibular head. The approach to determine the DTA differed between the two techniques. For the technique according to Jakob et al. [23, 26 , two circles were drawn in the slice $(1.0 \mathrm{~mm})$ proximal to the articular surface of tibia in the ankle joint, one circle was fitted into the tibia and one into the fibula in order to determine the respective centers. The DTA was drawn as a line connecting the two centers. For the technique according to Goutallier et al. [25], two tangents were placed to the articular aspects of the medial and the lateral malleolus one slice $(1.0 \mathrm{~mm})$ below the talar surface. The DTA was drawn as the line connecting the centers of the two tangents. For both techniques, the tibial torsion was calculated as the angle between the PTA and the DTA. All measurements were performed independently by one orthopedic surgeon and one radiologist (A.H. and M.M.). 


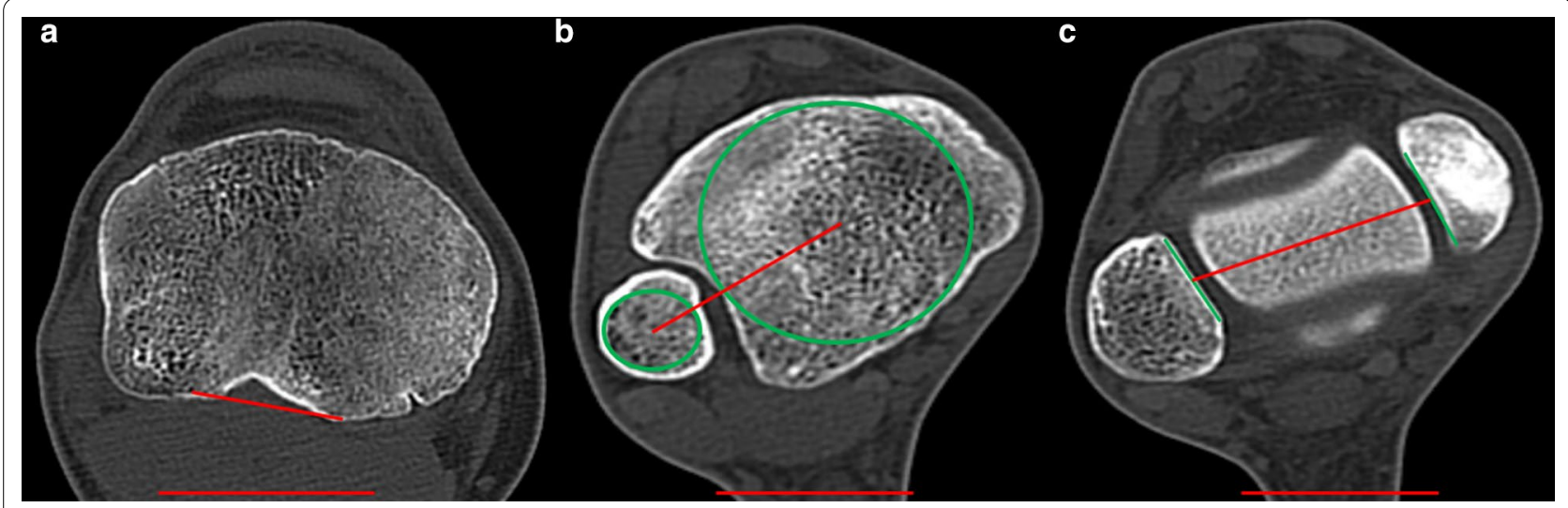

Fig. 2 2D measurement methods (a) proximal determination for both techniques (angle between red lines), (b) distal determination for technique 1, (c) distal determination for technique 2.-2D tibial torsion measures are calculated by the sum of angle between red lines in a plus angle between red lines in $\mathbf{b}$ for technique 1 and in $\mathbf{c}$ for technique 2

\section{Statistical analysis}

All data were documented with the REDCap software (Research Electronic Data Capture, Vanderbilt University, Nashville, Tennessee, USA). Statistical analyses were performed with SPSS (IBM SPSS Statistics 25, IBM, Armonk, New York, USA). Interobserver agreement was determined by calculating the intraclass correlation coefficients (ICC) based on a two-way random effects model assessing absolute agreement of single measures between readers. Agreement between different measurement methods was assessed using the ICC based on a two-way mixed model for absolute agreement of average measures generated. Differences of means between different methods were compared using paired samples $t$ test. Measurements for left and right legs as well as male and female patients were compared by applying independent samples $t$ tests to the means of the 2D and 3D methods, respectively. All data used for $t$ tests were tested for normality with a Shapiro-Wilk test. Mean absolute differences (MAD) were calculated. Tests were evaluated using a significance level of $p \leq 0.05$.

\section{Results}

Interobserver Agreement

Results for interobserver agreement are summarized in Table 1. The ICC for the Jakob and Goutallier methods were 0.917 (95\% CI $0.755-0.962)$ and 0.938 (95\% CI 0.905-0.959), respectively. For the 3D measurements, ICCs were 0.954 (95\% CI $0.930-0.970)$ and 0.95 (95\% CI $0.924-0.968$ ) for the 3D-TF and 3D-T methods, respectively.

\section{Intermethod Agreement}

All data were normally distributed. ICCs for intermethod agreement are summarized in Table 2. Agreement between the 2D methods was good $(0.873$ (95\% CI-0.115-0.968)), while 3D methods had excellent agreement (0.950 (95\% CI 0.860-0.977). Means were significantly different for both, 2D (30.1 vs. $24.1^{\circ}$ for Jakob and Goutallier, respectively, $p<0.001$, mean difference $6 \pm 2.6^{\circ}$, range $\left.0.3-12.7^{\circ}\right)$ and $3 \mathrm{D}$ methods ( 28.1 vs. $25.2^{\circ}$ for 3D-TF and 3D-T, respectively, $p<0.001$, mean difference $2.9 \pm 4.5^{\circ}$, range $-12.0-10.2$ ).

Agreement between 2 and 3D methods was moderate to good for all combinations. The Jakob method had ICCs of 0.795 (95\% CI $0.681-0.868)$ and 0.715 (95\% CI 0.481-0.834) when compared with 3D-TF and 3D-T, respectively. The Jakob method mean was also significantly different to both $3 \mathrm{D}-\mathrm{TF}$ and $3 \mathrm{D}-\mathrm{T}$ (30.1 vs. $28.1^{\circ}$ $(p=0.033)$, mean difference $2.0 \pm 8.5^{\circ}$ (range $-23.5-$ $\left.24.6^{\circ}\right)$ and 30.1 vs. $25.2^{\circ}(p<0.001)$, mean difference $4.9 \pm 9.6^{\circ}\left(\right.$ range $\left.-16.4-26.3^{\circ}\right)$.

Table 1 Correlation coefficients of interobserver agreement for all four methods

\begin{tabular}{lcr}
\hline Interobserver agreement 2D & Jakob method & Goutallier method \\
\hline ICC $(95 \% \mathrm{Cl})$ & $0.917(0.755-0.962)$ & $0.938(0.905-0.959)$ \\
\hline Interobserver agreement 3D & 3D-TF & 3D-T \\
\hline ICC $(95 \% \mathrm{Cl})$ & $0.954(0.963-0.985)$ & $0.950(0.924-0.968)$ \\
\hline
\end{tabular}

ICC intraclass correlation coefficient, Cl confidence interval, 3D-TF 3D method considering tibia and fibula, 3D-T 3D method only considering tibia 
Table 2 Intermethod agreement for all combinations between the four tested methods

\begin{tabular}{|c|c|c|}
\hline Intermethod agreement & ICC (95\% CI) & Mean value, $t$ test \\
\hline Jakob vs. Goutallier & $0.996(0.994-0.998)$ & $\begin{array}{l}30.1 \text { vs. } 24.1^{\circ}(p<0.001) \\
\text { MD: } 5.9 \pm 2.6^{\circ}\left(\text { range } 0.3-12.7^{\circ}\right)\end{array}$ \\
\hline Jakob vs. 3D-TF & $0.783(0.684-0.853)$ & $\begin{array}{l}30.1 \text { vs. } 28.1^{\circ}(p=0.033) \\
\text { MD: } 2.0 \pm 8.5^{\circ}\left(\text { range }-23.5-24.6^{\circ}\right)\end{array}$ \\
\hline Jakob vs. 3D-T & $0.715(0.481-0.834)$ & $\begin{array}{l}30.1 \text { vs. } 25.2^{\circ}(p<0.001) \\
\text { MD: } 4.9 \pm 9.6^{\circ}\left(\text { range }-16.4-26.3^{\circ}\right)\end{array}$ \\
\hline Goutallier vs. 3D-TF & $0.795(0.681-0.868)$ & $\begin{array}{l}24.1 \text { vs. } 28.1(p<0.001) \\
\text { MD: }-3.9 \pm 8.7^{\circ}\left(\text { range }-31.6-16.6^{\circ}\right)\end{array}$ \\
\hline Goutallier vs. 3D-T & $0.729(0.581-0.825)$ & $\begin{array}{l}24.1 \text { vs. } 25.2(p=0.348) \\
-1.0 \pm 9.9^{\circ}\left(\text { range }-24.5-19.9^{\circ}\right)\end{array}$ \\
\hline 3D-TF vs. 3D-T & $0.950(0.860-0.977)$ & $\begin{array}{l}28.1 \text { vs. } 25.2^{\circ}(p<0.001) \\
\text { MD: } 2.9 \pm 4.5^{\circ}\left(\text { range }-10.2-12.0^{\circ}\right)\end{array}$ \\
\hline
\end{tabular}

ICC intraclass correlation coefficients, $C I$ confidence interval, $M D$ mean difference

The ICCs for the Goutallier method were calculated to be 0.746 (95\% CI $0.558-0.847)$ and 0.729 (95\% CI $0.581-0.825)$. Its mean was significantly different to the 3D-TF method (24.1 vs. $28.1(p<0.001)$, mean difference $-3.9 \pm 8.7^{\circ}\left(\right.$ range $\left.\left.-31.6-16.6^{\circ}\right)\right)$, but not to the 3D-T method (24.1 vs. $25.2(p=0.348)$, mean difference $-1.0 \pm 9.9^{\circ}\left(\right.$ range $\left.\left.-24.5-19.9^{\circ}\right)\right)$.

\section{Left vs. Right and Female vs. Male}

Results regarding differences between side and genders are summarized in Table 3. All data were normally distributed. Torsion values for left and right legs did not differ significantly in $2 \mathrm{D}$ as well as in $3 \mathrm{D}(26.2 \pm 8.2 \mathrm{vs}$ $28.5 \pm 9.1^{\circ} \quad(p=0.232)$ and $27.2 \pm 12.8$ vs. $25.9 \pm 10.6^{\circ}$ $(p=0.632))$. The same is true for the differences between male and female in $2 \mathrm{D}\left(26.2 \pm 8.7\right.$ vs. $29.6 \pm 8.0^{\circ}$ $(p=0.115))$; it was however significantly different when assessed in $3 \mathrm{D}\left(25.0 \pm 10.8\right.$ vs. $\left.31.2 \pm 13.7^{\circ}(p=0.036)\right)$.

\section{Discussion}

Rotational malalignment of the lower extremities has received considerable attention in the last two decades, not only in children, but also in adults. However, the vast majority of studies have focused on abnormal femoral torsion and its role in femoroacetabular impingement, developmental dysplasia of the hip, patellofemoral disorders and other diseases [36-42]. Maltorsion of the tibia in adults remains poorly investigated. Nevertheless, there is certain knowledge about the negative clinical outcome in patients with a decreased or increased tibial torsion [11-16]. In addition, the lack of standardized measurement methods may have resulted in too little attention being paid to the relevance of tibial torsion.

The most important finding of this study is that the newly developed three-dimensional measurement technique showed a high interobserver reliability. The technique is capable of delivering values for the tibial torsion both with or without consideration of the fibula position.

The interobserver reliability of the $2 \mathrm{D}$ measurement techniques was slightly lower than for the 3D methods, achieving similar values as in the current literature [27]. The interclass correlation coefficients comparing the $3 \mathrm{D}$ technique with the two 2D measurement techniques for the tibial torsion were only moderate to good. However, a lower ICC does not necessarily implicate limited suitability of the methods. In this case, the differences might be explained by the diverging definition of the proximal tibial axis in the proposed 3D techniques. The 2D measurement methods define the proximal axis as the tangent to the dorsal cortex of the tibial head, but the kinematic relevance of the dorsal rim of the tibial bone is questionable. The $3 \mathrm{D}$ technique proposed in our paper, on the other hand, uses the part of the tibial plateau which is in contact with the femoral condyles to define the PTA.

Table $3 \boldsymbol{t}$ tests for side and gender differences of tibial torsion values

\begin{tabular}{lll}
\hline Left vs. right & 2D & 3D \\
\hline$t$ test $(p$ value $)$ & $26.2 \pm 8.2$ vs. $28.5 \pm 9.1^{\circ}(p=0.232)$ & $27.1 \pm 12.8$ vs. $25.8 \pm 10.5^{\circ}(p=0.632)$ \\
\hline Male vs. female & 2D & 3D \\
\hline$t$ test & $26.2 \pm 8.7$ vs. $29.6 \pm 8.0^{\circ}(p=0.115)$ & $25.0 \pm 10.8$ vs. $31.2 \pm 13.7^{\circ}(p=0.036)$ \\
\hline
\end{tabular}


Since a holistic 3D analysis with consideration of the kinematically relevant structures is desirable, we believe that this is a more suitable representation of the proximal tibial axis.

Since cross-sectional imaging has become standard in preoperative assessment of many orthopedic procedures, several studies were conducted to describe techniques for torsion measurements of the tibia based on 2D CT or MR images [23-28]. All proposed methods still used a twodimensional technique applied on selected slices at predefined anatomical positions of the tibia. None of them considers the three-dimensional anatomical constitution of the tibia. Fürmetz et al. proposed a three-dimensional assessment of the lower extremity as a whole [43]. In their work, tibial torsion was determined by the two most dorsal proximal points of the tibia and the two outermost points at the height of the ankle joint, a technique adapted from Liodakis et al. [27]. Even if calculated on the basis of three-dimensional landmark coordinates, their measurement technique only uses four points to define the torsion of a volumetric body. An interesting approach was proposed by Borish et al. where tibial torsion was measured using a capture motion system [44]. This is valuable with the increasing possibilities for dynamic analyses. However, some inaccuracy remains as the system uses surface markers that do not accurately reflect the anatomical landmarks of the tibia. The system is also not available in many facilities. However, we think that such a complex problem requires a technique which considers the whole three-dimensional surface of the tibial bone. Our method meets this requirement since a volumetric model is used for the calculation and it is therefore closer to the anatomic reality than points selected by the examiner. The fact that the method works with or without consideration of the fibula is convenient, since the position of the fibula is variable [24]. However, since the fibula is a relevant stabilizer for the ankle joint and makes a relevant contribution to the ankles kinematic function, we think it should be included in the analysis. However, the fibula can be excluded if it shows posttraumatic or postoperative alterations that make it unreliable.

A limitation of our study is that CT scans are not a first line diagnostic tool in every patient and segmentation is time-consuming and requires specific knowledge, which is not always accessible. However, modern CT technology has resulted in a substantially lower radiation dose, and future algorithms will be able to automatically apply 3D measurement methods to segment 3D models. 3D measurement methods, like the one presented in this paper, will be increasingly implemented in the surgery planning workflow in the coming years. Moreover, a robust measurement method can provide the basis for biomechanical studies investigating the role of tibial torsion with state-of-the-art techniques, such as movement and gait analysis.

\section{Conclusion}

The novel 3D measurement technique shows a high interobserver agreement and offers an applicable opportunity to assess the individual three-dimensional anatomy for assessing tibial torsion. As three-dimensional planning becomes successively more important for many surgical procedures, our measurement technique can provide the basis for the planning workflow in $3 \mathrm{D}$ as well as for future studies aiming for improved understanding of the tibial torsion and its role in healthy and pathological biomechanics.

\section{Abbreviations}

2D: two-dimensional; 3D: three-dimensional; CT: computed tomography; CTC : condylar tip clusters; DDH: developmental dysplasia of the hip; DTA: distal tibial axis; DTJP: distal tibial joint plane; ICC: intraclass correlation coefficient; MAD: mean absolute differences; $\mathrm{mm}$ : millimeters; MR: magnetic resonance; PCA: principal component analysis; PTA: proximal tibial axis; T: tibia; TF: tibia and fibula; TFCA: tibiofibular contact area; TPP: tibial plateau plane.

\section{Acknowledgements}

This work is part of the LEDECO project supported by the SNF Swiss National Science Foundation (Grant Number: 320030_182352).

\section{Author's contributions}

$\mathrm{AH}$ helped in conceptualization, methodology, investigation, writing-original draft, visualization, TR contributed to conceptualization, methodology, software, formal analysis, visualization, MM investigated the study, PF helped in resources, supervision, SF and RS helped in resources, supervision, writingreview and editing. All authors read and approved the final manuscript.

\section{Funding}

There is no funding source.

\section{Availability of data and materials}

The datasets used and/or analyzed during the current study are available from the corresponding author on reasonable request.

Ethical approval and consent to participate

The local ethical committee approved this study (Zurich Cantonal Ethics Commission, 2017-01,616).

\section{Consent for publication}

All patients gave their informed consent for their participation in and the publication of this study.

\section{Competing interests}

The authors declare that they have no competing interests.

\section{Author details}

${ }^{1}$ Department of Orthopaedics, Balgrist University Hospital, University of Zurich, Forchstrasse 340, 8008 Zurich, Switzerland. ${ }^{2}$ Research in Orthopaedic Computer Science, Balgrist University Hospital, University of Zurich, Zurich, Switzerland. ${ }^{3}$ Balgrist University Hospital, University of Zurich, RadiologyZurich, Switzerland.

Received: 10 November 2020 Accepted: 29 December 2020

Published online: 15 February 2021 


\section{References}

1. Le Damany PGM (1903) Les torsions osseuses, leur role dans la transformation des membres. J Anat Physiol. J Anatom Physiol 39:246-450

2. Eckhoff DG, Winter WG (1994) Femoral and tibial torsion. Clin Orthop Relat Res 302:2-3

3. Le Damany PGM (1909) La torsion du tibia, normale, pathologique, expérimentale. Journal de l'Anatomie et Physiologie 45:598-615

4. Hutter CG Jr, Scott W (1949) Tibial torsion. J Bone Joint Surg Am 31A(3):511-518

5. Staheli LT, Engel GM (1972) Tibial torsion: a method of assessment and a survey of normal children. Clin Orthop Relat Res 86:183-186

6. Staheli LT (1977) Torsional deformity. Pediatr Clin North Am 24(4):799-811

7. Staheli LT (1983) In-toeing and out-toeing in children. J Fam Pract 16(5):1005-1011

8. Staheli LT, Corbett M, Wyss C, King H (1985) Lower-extremity rotational problems in children. Normal values to guide management. J Bone Joint Surg Am. 67(1):39-47

9. Yoshioka Y, Siu DW, Scudamore RA, Cooke TD (1989) Tibial anatomy and functional axes. J Orthop Res 7(1):132-137

10. Kling TF Jr, Hensinger RN (1983) Angular and torsional deformities of the lower limbs in children. Clin Orthop Relat Res 176:136-147

11. Kyro A (1997) Malunion after intramedullary nailing of tibial shaft fractures. Ann Chir Gynaecol 86(1):56-64

12. Puloski S, Romano C, Buckley R, Powell J (2004) Rotational malalignment of the tibia following reamed intramedullary nail fixation. J Orthop Trauma 18(7):397-402

13. Svoboda SJ, McHale K, Belkoff SM, Cohen KS, Klemme WR (2002) The effects of tibial malrotation on the biomechanics of the tibiotalar joint. Foot Ankle Int 23(2):102-106

14. Turner MS, Smillie IS (1981) The effect of tibial torsion of the pathology of the knee. J Bone Joint Surg Br. 63-B(3):396-398

15. van der Schoot DK, Den Outer AJ, Bode PJ, Obermann WR, van Vugt AB (1996) Degenerative changes at the knee and ankle related to malunion of tibial fractures. 15-year follow-up of 88 patients. J Bone Joint Surg Br 78(5):722-725

16. Hicks J, Arnold A, Anderson F, Schwartz M, Delp S (2007) The effect of excessive tibial torsion on the capacity of muscles to extend the hip and knee during single-limb stance. Gait Posture 26(4):546-552

17. Dejour H, Walch G, Nove-Josserand L, Guier C (1994) Factors of patellar instability: an anatomic radiographic study. Knee Surg Sports Traumato Arthrosc 2(1):19-26

18. Duparc F, Thomine JM, Simonet J, Biga N (2014) Femoral and tibial bone torsions associated with medial femoro-tibial osteoarthritis. Index of cumulative torsions. Orthop Traumatol Surg Res 100(1):69-74

19. Takai S, Sakakida K, Yamashita F, Suzu F, Izuta F (1985) Rotational alignment of the lower limb in osteoarthritis of the knee. Int Orthop 9(3):209-215

20. Terjesen T, Benum P, Anda S, Svenningsen S (1982) Increased femoral anteversion and osteoarthritis of the hip joint. Acta Orthop Scand 53(4):571-575

21. Tonnis D, Heinecke A (1999) Acetabular and femoral anteversion: relationship with osteoarthritis of the hip. J Bone Joint Surg Am 81(12):1747-1770

22. Clementz BG (1989) Assessment of tibial torsion and rotational deformity with a new fluoroscopic technique. Clin Orthop Relat Res 245:199-209

23. Jakob RP, Haertel M, Stussi E (1980) Tibial torsion calculated by computerised tomography and compared to other methods of measurement. J Bone Joint Surg Br. 62-B(2):238-242

24. Madadi F, Madadi F, Maleki A, Shamie AN, Washington ER 3rd, Yazdanshenas H (2016) A new method for tibial torsion measurement by computerized tomography. J Orthop 13(1):43-47

25. Goutallier D, Van Driessche S, Manicom O, Sariali E, Bernageau J, Radier C (2006) Influence of lower-limb torsion on long-term outcomes of tibial valgus osteotomy for medial compartment knee osteoarthritis. J Bone Joint Surg Am 88(11):2439-2447

26. Waldt. Messverfahren und Klassifikationen in der muskuloskelettalen Radiologie. 2011.
27. Liodakis E, Doxastaki I, Chu K et al (2012) Reliability of the assessment of lower limb torsion using computed tomography: analysis of five different techniques. Skeletal Radiol 41(3):305-311

28. Jend H-H, Heller M, Dallek M, Schoettle H (1981) Measurement of tibial torsion by computer tomography. Acta Radiol Diagn (Stockh) 22(3A):271-276

29. Furnstahl P, Vlachopoulos L, Schweizer A, Fucentese SF, Koch PP (2015) Complex osteotomies of tibial plateau malunions using computerassisted planning and patient-specific surgical guides. J Orthop Trauma 29(8):e270-e276

30. Jentzsch T, Vlachopoulos L, Furnstahl P, Muller DA, Fuchs B (2016) Tumor resection at the pelvis using three-dimensional planning and patientspecific instruments: a case series. World J Surg Oncol 14(1):249

31. Vlachopoulos L, Schweizer A, Meyer DC, Gerber C, Furnstahl P (2016) Three-dimensional corrective osteotomies of complex malunited humeral fractures using patient-specific guides. J Shoulder Elbow Surg 25(12):2040-2047

32. Fürnstahl P, Schweizer A, Graf M et al (2016) Surgical treatment of long-bone deformities: 3D preoperative planning and patient-specific instrumentation. In: Zheng G, Li S (eds) Computational radiology for orthopaedic intervensions 23. Springer, Cham

33. Forgy $E$ (1965) Cluster analysis of multivariate data: efficiency versus interpretability of classification. Biometrics 21(3):768-769

34. Arthur D, Vassilvitskii S (2007) k-means++: the advantages of careful seeding. In: SODA 2007: proceedings of the eighteenth annual ACMSIAM symposium on Dicrete algorithms. pp 1027-1035.

35. Schneider P, Eberly DH (2002) Geometric tools for computer graphics. Elsevier, Amsterdam

36. Schmaranzer F, Lerch TD, Siebenrock KA, Tannast M, Steppacher SD (2019) Differences in femoral torsion among various measurement methods increase in hips with excessive femoral torsion. Clin Orthop Relat $\mathrm{R}$ 477(5):1073-1083

37. Mascarenhas WV, Rego P, Dantas P, Caetano AP, Jans L, Sutter R et al (2018) Can we discriminate symptomatic hip patients from asymptomatic volunteers based on anatomic predictors? A 3-dimensional magnetic resonance study on cam, pincer, and Spinopelvic parameters. Am J Sports Med 46(13):3097-3110

38. Sankar WN, Neubuerger CO, Moseley CF (2009) Femoral anteversion in developmental dysplasia of the hip. J Pediatr Orthop 29(8):885-888

39. Fritz B, Bensler S, Leunig M, Zingg PO, Pfirrmann CWA, Sutter R (2018) MR assessment of supra- and infratrochanteric femoral torsion: association with femoroacetabular impingement and hip dysplasia. AJR Am J Roentgenol 211(1):155-161

40. Sutter R, Dietrich TJ, Zingg PO, Pfirrmann CWA (2012) Femoral antetorsion: comparing asymptomatic volunteers and patients with femoroacetabular impingement. Radiology 263(2):475-483

41. Imhoff FB, Cotic M, Liska F, Dyrna FGE, Beitzel K, Imhoff AB et al (2019) Derotational osteotomy at the distal femur is effective to treat patients with patellar instability. Knee Surg Sports Traumatol Arthrosc 27(2):652-658

42. Kamath AF, Ganz R, Zhang H, Grappiolo G, Leunig M (2015) Subtrochanteric osteotomy for femoral mal-torsion through a surgical dislocation approach. J Hip Preserv Surg 2(1):65-79

43. Furmetz J, Sass J, Ferreira T, Jalali J, Kovacs L, Muck F et al (2019) Threedimensional assessment of lower limb alignment: accuracy and reliability. Knee 26(1):185-193

44. Borish CN, Mueske NM, Wren TAL (2017) A comparison of three methods of measuring tibial torsion in children with myelomeningocele and normally developing children. Clin Anat 30(8):1043-1048

\section{Publisher's Note}

Springer Nature remains neutral with regard to jurisdictional claims in published maps and institutional affiliations. 